\title{
Quantum State Transfer among Crystallographic Groups of N-V Centers in Diamond
}

\author{
Özgür E. Müstecaplığlu \\ Department of Physics, Koç University, Sariyer, İstanbul, 34450, Turkey
}

\begin{abstract}
We investigate collections of Nitrogen-Vacancy (N-V) Centers in diamond crystals coupled to a circuit QED system of a coplanar waveguide (CPWG) resonator. Our analysis reveals that different symmetry axes oriented $\mathrm{N}-\mathrm{V}$ centers in the diamond host can be grouped into bosonic modes of collective quasi-spin wave excitations so that the hybrid system can be described as an analog of an exciton-polariton type cavity QED model. We examine such model for quantum state transfer among distinct crystallographic groups of N-V centers in a single diamond as well as two spatially distant diamonds. Rabi oscillations, mode entanglement, possible use of N-V classes as spin ensemble qubits and an implementation of continuous-time quantum random walk are discussed.
\end{abstract}

Keywords: Color centers, vacancies and other defects, N-V centers in diamond, circuit QED, hybrid systems, quantum communication and computation

\section{INTRODUCTION}

Recent demonstrations of electronic spin ensembles, such as the electron spin of nitrogen atoms in fullerene cages and phosphorous donors in silicon, ${ }^{1}$ as well as $\mathrm{P} 1^{2}$ and N-V centers in diamonds, ${ }^{3}$ strongly coupled to microwave photons promise efficient implementation of quantum memories. Further progress of such hybrid systems, ${ }^{4}$ in particular developments to couple them from microwave to visible domain would make them ideal compact and complete devices for quantum computation and communication applications. ${ }^{5}$

To reveal the full quantum optical potential of $\mathrm{N}-\mathrm{V}$ centers, we first develop a general quantum optical model taking into account all the four distinct crystallographic classes for them. N-V centers coupled to superconducting coplanar waveguide resonators are investigated for quantum state transfer among their four distinct crystallographic groups. We find that by adjusting a bias magnetic field orientation, state transfer among the crystallographic groups can be achieved. Rabi oscillations among the basis of such crystallographic classes is found. This allows for possible use of the crystallographic classes as spin ensemble qubits within a single diamond. Alternatively, we also discuss state transfer protocols between spatially distinct diamonds with N-V centers including all crystallographic classes. We argue that such a diamond network coupled with microwave cavity photons acting in between can be used as a quantum random walk system. In addition we discuss both local and distant entanglement of such crystallographic classes. Finally we also argue how to use various measurements of fidelities can be exploited to determine population distribution among the crystallographic classes.

Our methodology is based upon employing a statistical mutation transformation of the usual spin Hamiltonian for the case of a large ensemble of $\mathrm{N}-\mathrm{V}$ centers. Dominant Zeeman interaction with the external bias magnetic field and the microwave cavity field is then mutated from a spin model to a fully bosonic model, similar to coupled cavities. Due to the presence of distinct crystallographic groups however the effective model resembles coupled multimode cavities. Using standard quantum optical methods after that, we determine the dark modes of the effective cavity model. The coupling between various crystallographic classes is mediated by the microwave cavity photon so that the system in fact consists of atom-molecules or strictly speaking N-V center polaritons. Our model can be treated analytically and we find simple intuitive expressions which is further analyzed to extract additional information to determine initial population distribution among the crystallographic classes from post fidelity measurements.

E-mail: omustecap@ku.edu.tr, Telephone: 902123381424

Integrated Photonics: Materials, Devices, and Applications, edited by Ali Serpengüzel, Giancarlo C. Righini, Alfred Leipertz, Proc. of SPIE Vol. 8069, 80690E · C 2011 SPIE · CCC code: 0277-786X/11/\$18 · doi: 10.1117/12.887122 


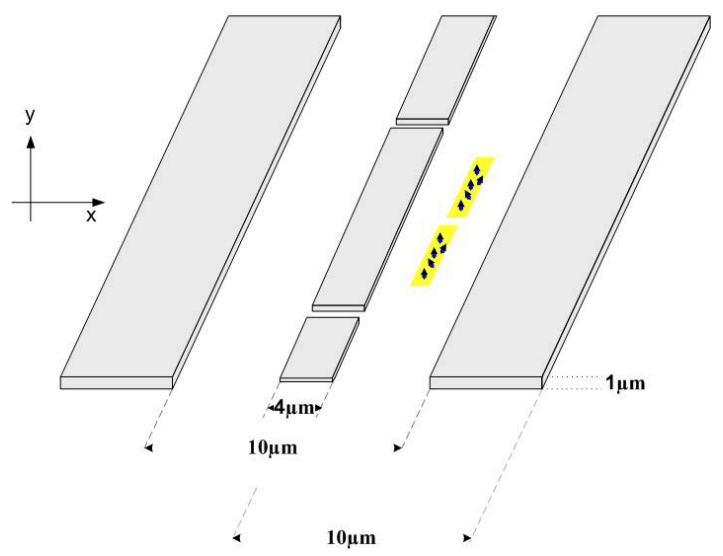

Figure 1. Geometry of the hybrid system consisting of the CPWG resonator containing two N-V center ensembles at the adjacent antinodes of the magnetic field of the resonator mode.

\section{HYBRID SYSTEM}

The system under consideration is a hybrid of a solid state resonator and two molecular sub-systems. Molecular ensembles are formed in two diamond crystals with N-V color center defects placed at the adjacent antinodes of the magnetic field of the CPWG resonator mode as shown in Fig. 1. Dimensions of the system are chosen in parallel to the available experimental systems ${ }^{6-8}$ and in accordance with the physical requirements, that will be discussed in the subsequent sections, for efficient enhancement of the collective magnetic dipole coupling of the ensembles through the cavity mode. Following subsections describe the components of the hybrid system in detail.

\subsection{CPWG Resonator}

Coplanar waveguide resonator is a special one-dimensional superconducting transmission line resonator (TLR) depicted in Fig. 1. It is made from a central microstripline of length $L$ in between two ground half-plates. A one-dimensional Fabry-Perot TLR is formed due to the capacitive coupling to input and output ports. For a TLR of length $L$ and effective dielectric constant $\epsilon_{e f f}$, resonator frequency of mode $n$ is independent of the cavity width $\omega_{n} / 2 \pi=n c_{0} / 2 L \sqrt{\epsilon_{e f f}}$, with $c_{0}$ being the speed of light in vacuum. One-dimensional cavities offer strongly reduced mode volume for enhanced cavity-sample coupling. $\epsilon_{\text {eff }}$ lies between the substrate dielectric constant $\epsilon_{r}$ and 1. Its exact expression can be determined by conformally mapping the CPWG into rectangular geometry. ${ }^{9}$

Mode profile of the cavity is determined by assuming the cavity is shielded by metallic walls. The length of the cavity is $L=\lambda=25 \mathrm{~mm}$. Total transverse width of the system is $W=10 \mu \mathrm{m}$. The central conductor and the grounding planes respectively are $4 \mu \mathrm{m}$ and $5 \mu \mathrm{m}$. The substrate is GaAs with the dielectric constant $\epsilon_{s}=12.9$, and of depth $H=5 \mu \mathrm{m}$. Central conductor is assumed to operate at the single photon regime. Quasi-static quasiTEM modes are found by solving the Laplace equation with the metallic boundary conditions. Fringe effects and other technical complications can be studied computationally by finite element method (FEM) solvers which are available commercially. ${ }^{10}$ For our purposes details of the field distributions near the boundaries are not required.

Another influence of the boundaries is the nonlinear response of the circuit. ${ }^{11,12}$ Due to small cross section of the transmission line and inhomogeneous current distribution along it, distributed inductance can have current dependent contribution. We wish to operate the cavity in single photon regime so that weak driving powers will be sufficient, as such nonlinear response can be ignored. Employing the numerical analysis method based upon Gaussian elimination technique developed for conductor backed and shielded CPWGs, ${ }^{13}$ the usual charge 
field quantization of the distributed circuit model of the $\mathrm{TLR}^{14-16}$ can be translated to quantized electric and magnetic field operators.

Striking analogies between quantized lumped circuits and cavity QED systems interacting with spins has been recognized long ago. ${ }^{17,18}$ There are still some challenges in quantization of lossy circuits and lossy TLRs. ${ }^{19,20}$ Due to the presence of input and output semi-infinite channels, the actual cavity is an open system. It has been rigorously established that cavity mode can be reliably considered to be a quantum object in a TLR, as long as the precision of the experiment is below the standard quantum limit which includes both the cavity mode and the reservoir fluctuations. ${ }^{20}$ In the subsequent discussions, input and output reservoirs shall be ignored. Following the charge-flux fields canonical quantization scheme for a lossless TLR ${ }^{21}$ (the alternative of number-phase fields quantization ${ }^{22}$ may face quantum phase controversy and more suitable for Josephson Junction qubits), an equivalent circuit Lagrangian density for the TLR, defined over $[-L / 2, L / 2]$ interval, can be written as

$$
\mathcal{L}\left(\dot{Q}, Q^{\prime}\right)=\frac{l}{2}\left(\frac{\partial Q}{\partial t}\right)^{2}-\frac{1}{2 c}\left(\frac{\partial Q}{\partial z}\right)^{2},
$$

where $l, c$ are the distributed inductance and capacitance density of the TLR. Generalized Euler-Lagrange equations derived from the Hamilton's Principle leads to usual Telegraphist's equations that can be combined into a linear wave equation with wave speed parameter $v=1 / \sqrt{l c}$ for the charge field $Q$. Solving the wave equation by separation of variables gives, under periodic boundary conditions and assuming charge neutrality, spatial profile of the axial distribution of the charge field in terms of even $\cos (j \pi z / L)(j$ : odd integer) and odd $\sin (j \pi z / L)(j$ : even integer) modes. Time dependent part of the charge field is canonically quantized in terms of set of harmonic oscillator modes. From the quantized charge field $\hat{Q}$, distribution of the quantized voltage field $\hat{V}$ along the TLR can be found from $\hat{V}=(1 / c)(\partial \hat{Q} / \partial z)$, which is

$$
\begin{aligned}
\hat{V} & =-\sum_{j=1,3, \ldots} \sqrt{\frac{\hbar \omega_{j}}{L c}}\left(a_{j} \mathrm{e}^{-\mathrm{i} \omega_{j} t}+a_{j}^{\dagger} \mathrm{e}^{\mathrm{i} \omega_{j} t}\right) \sin \frac{j \pi z}{L} \\
& +\sum_{j=2,4, \ldots} \sqrt{\frac{\hbar \omega_{j}}{L c}}\left(a_{j} \mathrm{e}^{-\mathrm{i} \omega_{j} t}+a_{j}^{\dagger} \mathrm{e}^{\mathrm{i} \omega_{j} t}\right) \cos \frac{j \pi z}{L}
\end{aligned}
$$

In order to take into account quasi-1D nature of the TLR, a cutoff can be introduced to the upper limit of the summations.

The potential field found above is one of the boundary conditions that is used to determine quasi-TEM mode. In the single photon regime we take the rms value of the potential $V_{0}=\sqrt{\hbar \omega_{c} / 2 L c}$ on the central conductor. Using $\epsilon_{\text {eff }} \sim 1$, the fundamental cavity mode frequency becomes $\omega_{c} / 2 \pi \sim 6 \mathrm{GHz}$. Taking the cavity capacitance $C=L c=1 \mathrm{pF}$, we find $V_{0} \sim 1 \mu \mathrm{V}$ so that single photon magnetic field strength can be estimated to be in the order of few $\mu \mathrm{G}$, which is consistent with our numerical evaluations. The electric and magnetic fields are determined from the solution of the Laplace equation for a scalar potential $U$ by $\vec{E}=-\nabla U$ and $H=(\hat{z} \times \vec{E}) / Z$ with $Z$ being the impedance of the medium. Following the semi-analytical solution of CPWG modes, ${ }^{13}$ the potential above $\left(U_{+}\right)$and below $\left(U_{-}\right)$the substrate surface is written as

$$
\begin{aligned}
& U_{+}=\sum_{i=1}^{\infty} A_{i} \mathrm{e}^{-(2 i-1) \pi y / W} \cos \left((2 i-1) \frac{\pi x}{W}\right) \\
& U_{-}=\sum_{i=1}^{\infty} B_{i} \sinh \left((2 i-1) \frac{\pi y}{W}\right) \cos \left((2 i-1) \frac{\pi x}{W}\right)
\end{aligned}
$$

Using the continuity of the potential at the surface of the substrate, of thickness $H$, we get the relation $A_{i}=$ $B_{i} \sinh ((2 i-1) \pi H / W)$. Continuity of the normal electric displacement in the slots at the substrate surface, together with the given potentials on the grounding and central conductors fix the coefficients $A_{i}$. On a discrete set of points $x_{j}$ at the surface, boundary conditions can be reduced into a linear system $M_{j i} A_{i}=U_{j}^{(s)}$ where $U_{j}^{(s)}$ 


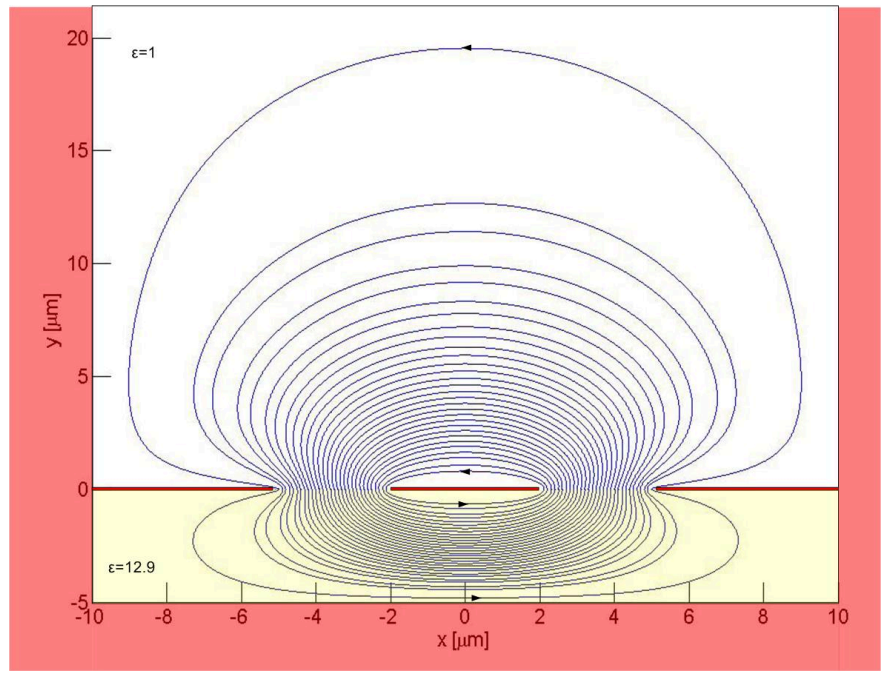

Figure 2. Magnetic field lines in a cross-section of the shielded CPWG system. Cross section is taken at a longitudinal antinode of the magnetic field.

represents the potentials such that $U_{j}^{(s)}=\hat{V}$ for the $x_{j}$ on the central conductor. While $x_{j}$ is out of the central conductor, $U_{j}^{(s)}=0$. Thus, solution of the linear system for $A_{i}$ translates the quantum character of the charge field into the electromagnetic mode through $\hat{V}$. These considerations lead to magnetic field of the fundamental cavity mode of the form

$$
\vec{B}=\frac{\mathrm{i}}{\sqrt{2}} \vec{B}_{0}(x, y)\left(\hat{a}_{c}-\hat{a}_{c}^{\dagger}\right) \cos \frac{\pi z}{L} .
$$

A replacement of $a_{1}=-\mathrm{i} a_{c}$ is used to make the result resemble usual cavity QED form of the quantized electromagnetic field. $\vec{B}_{0}(x, y)$ is determined numerically, by solving the linear equation system for $A_{i}$. Longitudinal behavior of $\vec{B}$ indicates that spin ensembles need to be $\lambda / 2$ apart in a cavity of length $L=\lambda$, by placing them at the adjacent magnetic field antinodes. On the other hand, the antinodes are at the cavity boundaries for the fundamental mode. To avoid the fringe effects, the samples need to be either shifted from the antinodes towards the cavity center or one can consider the second cavity mode and place the samples at the center of the slots where an antinode of the second mode lies. It is also necessary to have sufficiently small samples along the axial direction relative to the cavity length so that longitudinal variations in the coupling of individual spins to the cavity mode can be ignored. To investigate transverse variations that can arise due to the variations of the magnetic field along the gaps, we examine the numerically determined transverse mode profiles given in Fig. 2 .

Near the middle of the gaps, and towards the central conductor, the field is satisfactorily uniform in its direction. To examine the uniformity of its magnitude at the same region of the surface, field behavior is investigated by approaching to the surface as shown in Fig. 3.

Magnetic field magnitude approaches to a plateau as the distance to the substrate surface goes to zero. This plateau, though not at the field maximum, is the same region where the field direction is uniform, and hence provides an identical magnetic coupling zone for the ensemble spins. Having fixed the transverse and longitudinal coordinates to place the spin ensembles optimally, the number of spins that can be placed in such a zone can be estimated. This shall be done in the following sub-section, where N-V centers will be introduced.

\subsection{N-V Centers as Spin Ensembles}

We consider N-V color center point defects in diamond (see Fig. 4$)^{23}$ as the spin ensembles in the CPWG cavity. Synthetic, Nitrogen rich (Type-1b) thin diamonds may be produced by Chemical Vapor Deposition (CVD) technique. Alternatively, nanocrystalline powders may be considered. Irradiating the sample by high 


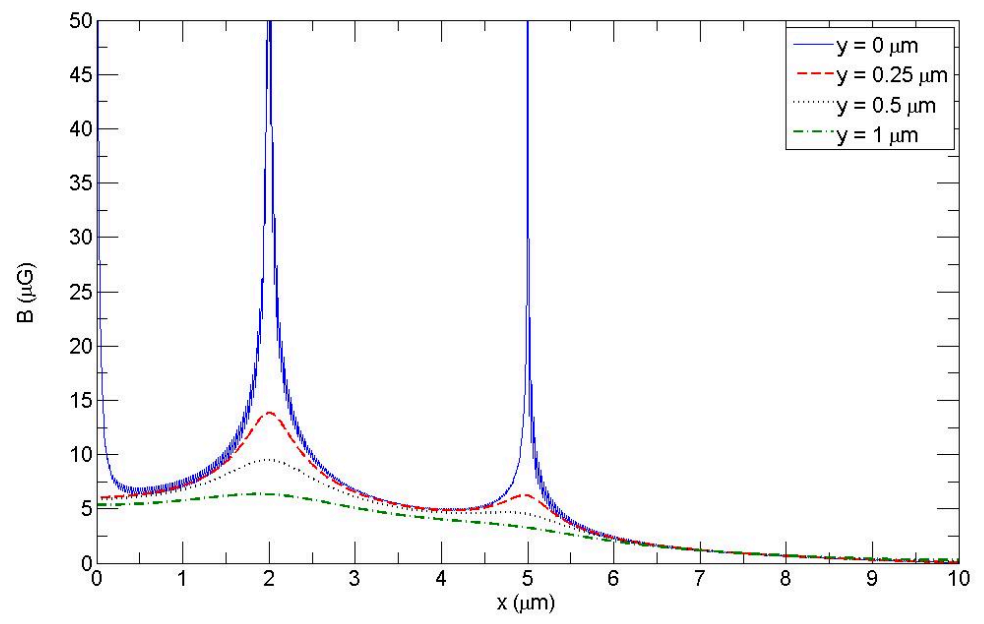

Figure 3. Magnitude of the magnetic field as it is probed at closer and closer distances to the substrate surface.

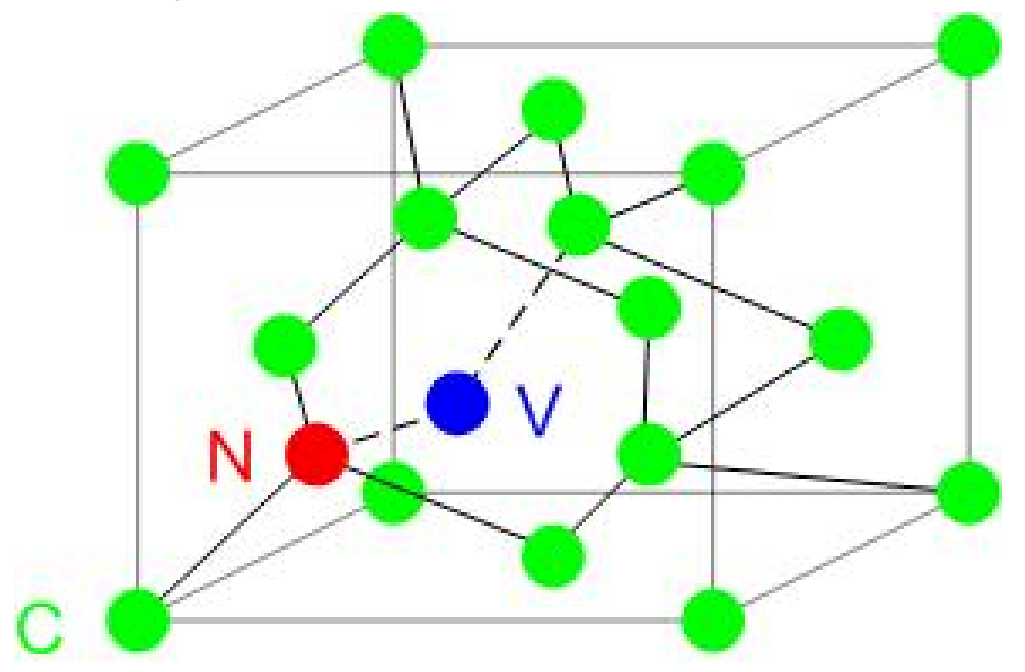

Figure 4. Structural model of the N-V center oriented in [111] direction in the diamond crystal cell. The centre is at a trigonal $\left(C_{3 v}\right)$ symmetry site.

dose of energetic electrons (with energies $>\mathrm{MeV}$ ) and subsequent annealing $\left(>1000^{\circ} \mathrm{C}\right.$ ), damage the sample with dense vacancies trapped near the nonaggregated, substitutional, deep nitrogen impurities. N-V center concentrations up to $10^{18} \mathrm{~cm}^{-3}$ are reported in the experiments. ${ }^{24}$ From Figs. 2-3, transverse height and width of the sample is chosen to be $1 \mu \mathrm{m}$ and $2 \mu \mathrm{m}$, respectively. Taking density of $\mathrm{N}-\mathrm{V}$ centers as $10^{17} \mathrm{~cm}^{-3}$, and length of the sample as $5 \mu \mathrm{m}$, total number of $\mathrm{N}-\mathrm{V}$ centers is found to be $N=10^{6}$. A cavity length $L=25 \mathrm{~mm}$ is assumed, being same with the fundamental mode wavelength $\lambda$.

Assuming such a type-1b diamond microcrystal can be made with high density of defects, it is also demanded that the sample should be isotropically purified to reduce ${ }^{13} \mathrm{C}$ impurities. Though not as serious as it can be for lower density of $\mathrm{N}-\mathrm{V}$ centers, it could be a source of dephasing. The major source of decoherence in high density $\mathrm{N}-\mathrm{V}$ centers would be ${ }^{14} \mathrm{~N}$ nuclei, if the efficiency of $\mathrm{N}-\mathrm{V}$ center creation is not perfect. On the other hand, some nearest nuclei (abundant ${ }^{13} \mathrm{C},{ }^{14} \mathrm{~N}$, or implanted ${ }^{15} \mathrm{~N}$ ) are shown to be beneficial for two qubit operations. ${ }^{25-28}$ In the present investigation, neither nuclear spin baths nor the single nuclear spin coupling shall be considered for simplicity. 
The excited states of the $\mathrm{N}-\mathrm{V}$ center are complicated due to spin-orbit, spin-spin and crystal strain influences. The ground state, however, is well established, ${ }^{29,30}$ it is a ${ }^{3} A$ electron spin triplet, paramagnetic system, which is explained by a six electron model, making the defect negatively charged $\mathrm{NV}^{-}$. The upper state doublet, $|X\rangle=\left|m_{s}=+1\right\rangle,|Y\rangle=\left|m_{s}=-1\right\rangle$, and the lower state singlet $|Z\rangle=\left|m_{s}=0\right\rangle$ are split from each other by $2.87 \mathrm{GHz}$ due to anisotropic dipolar interaction of two unpaired electrons in diamond crystal field. The ground state of a single $\mathrm{N}-\mathrm{V}$ center is described by the Hamiltonian

$$
H_{n v}=H_{z f}+H_{h f}+H_{q}+H_{n Z},
$$

where the hyperfine $H_{h f}$, quadrupole $H_{q}$ and nuclear Zeeman $H_{n Z}$ contributions shall be neglected in the present analysis.

$$
H_{z f}=S^{T} \tilde{D} S,
$$

is the zero-field Hamiltonian with $\tilde{D}$ is the zero-field splitting tensor and $S$ is the spin-1 vector. In the molecular body frame, which is the principal axes frame with the symmetry axis being the $\mathrm{N}-\mathrm{V}$ center orientation axis, $\tilde{D}$ is diagonal with $D_{x}=D_{y}=D_{\perp}, D_{z}=D_{\|}$. Thus we get (unless otherwise noted $\hbar=1$ is used in the following

$$
H_{z f}=D\left(S_{z}^{2}-\frac{1}{3} S(S+1)\right),
$$

with $D=3 D_{\|} / 2=2.87 \mathrm{GHz}$. The quantization axis is the same with the $\mathrm{N}-\mathrm{V}$ center alignment, thus in the spin basis $|m\rangle$ the zero-field Hamiltonian can be written, after dropping the constants, as

$$
H_{z f}=D \sum_{m} m^{2}|m\rangle\langle m| .
$$

\subsection{Coupling N-V centers to CPWG Resonator}

The samples are assumed to be placed over the substrate in one of the slots of the CPWG cavity such that the crystal axis $<110>$ is along the $y$-axis. ${ }^{31}$ Chosen sample sizes locations makes the magnetic field is uniform and in the $y$-direction, over the sample. $\mathrm{N}-\mathrm{V}$ center has four equivalent quantization axes $[111],[\overline{1}, \overline{1}, 1],[\overline{1} 1 \overline{1}]$ due to the $C_{3 v}$ symmetry, and $[1 \overline{1} \overline{1}]$ as shown in Fig. 5. In typical experiments, external magnetic fields are used to tune one among four possible crystallographic classes to couple microwave fields. ${ }^{32}$ This causes loss of three quarter of $\mathrm{N}-\mathrm{V}$ centers in the sample for possible collective enhancement of the coupling to the cavity. It has been noted that an intriguing approach to $\mathrm{N}-\mathrm{V}$ centers is to design methods to exploit all the four crystallographic classes of $\mathrm{N}-\mathrm{V}$ centers in magnetometry. ${ }^{32}$ Very recently, effect of a static magnetic field on the electron spin resonance of an ensemble of $\mathrm{N}-\mathrm{V}$ centers oriented in the four possible directions in a bulk diamond has been experimentally studied. ${ }^{33}$ These suggests that we have to carefully take into account different set of $\mathrm{N}-\mathrm{V}$ centers in the spin ensembles and furthermore to check if all $\mathrm{N}-\mathrm{V}$ centers can be exploited in the collective enhancement of the cavity coupling without any external detuning static magnetic fields.

In order to represent many $\mathrm{N}-\mathrm{V}$ centers in different crystallographic groups, it is convenient to introduce collective spin operators as analogs of Hubbard operators in strongly correlated systems as

$$
X_{f}^{m n}=\sum_{j=1}^{N_{f}}|m\rangle_{j}^{(f)(f)}\langle n| .
$$

Here, $f=1,2,3,4$ is the symmetry axis label, $j=1 . . N_{f}$ is the N-V center index, $N_{f}$ is the number of N-V centers aligned along the symmetry axis $f$, and $m, n=-1,0,1$ are the spin quantum numbers. In this notation, the total Hamiltonian becomes

$$
H_{z f}=D \sum_{m f} m^{2} X_{f}^{m m} .
$$




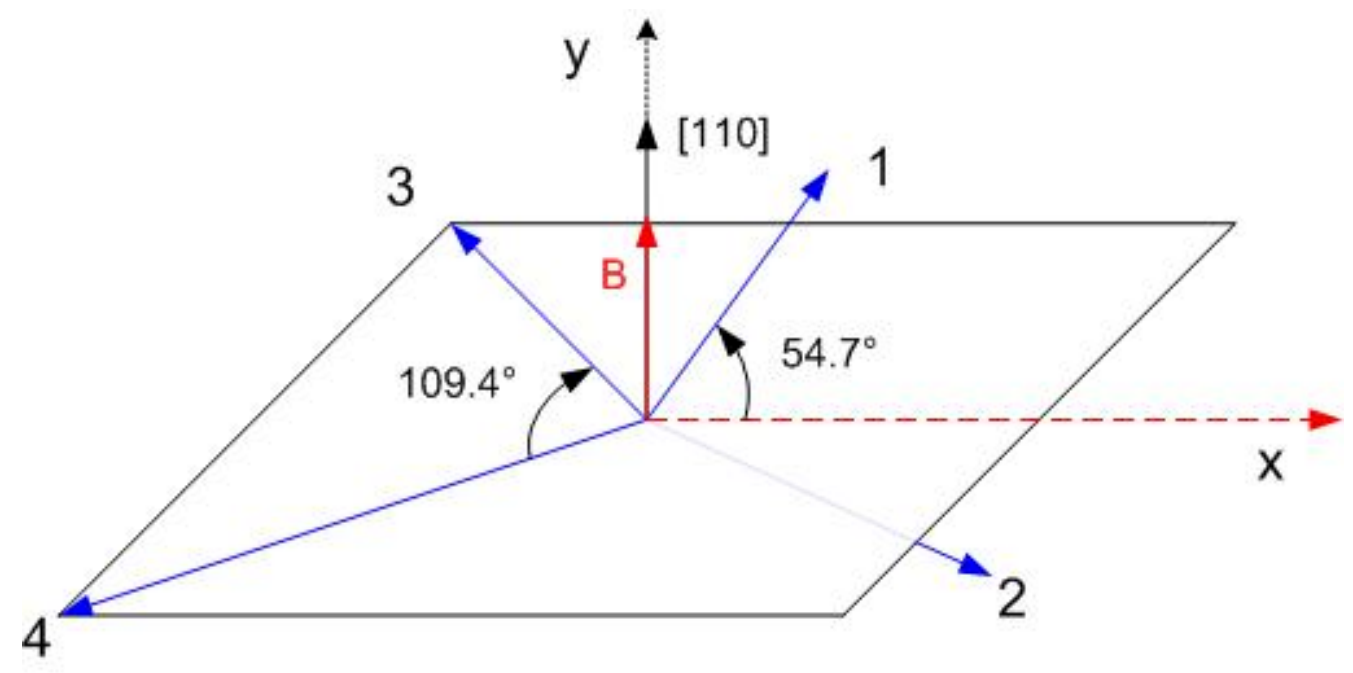

Figure 5. Equivalent quantization axes for N-V centers for a diamond crystal oriented in [110] direction. Magnetic field is in parallel with the crystal direction.

The N-V centers are coupled to the cavity through the electronic Zeeman interaction, which is $H_{e Z}=$ $\mu_{B} B^{T} g S$, for a single spin. In the molecular frame of $\mathrm{N}-\mathrm{V}$ center, $g$ is a diagonal tensor with $g_{\perp}=2.0024$ and $g_{\|}=2.0028$. The magnetic field over the spin ensemble at the surface is uniform and aligned in the $y$-direction so that we represent it in the lab frame as $\vec{B}=\hat{B} \hat{y}$, where

$$
\hat{B}= \pm \frac{\mathrm{i}}{\sqrt{2}} B_{0}\left(\hat{a}_{c}-\hat{a}_{c}^{\dagger}\right) .
$$

The sign difference $( \pm)$ in $\hat{B}$ is due to the placement of the spin ensembles in two adjacent antinodes separated by $L / 2(L=\lambda)$. Writing $\vec{B}$ in four different molecular frames in Fig. 5 , cavity field coupling of N-V centers belonging to different crystallographic classes can be determined. In many-N-V center system, the total interaction Hamiltonian for one of the spin ensembles becomes

$$
H_{\mathrm{int}}=\sum_{f} H_{\mathrm{int}}^{(f)},
$$

where

$$
H_{\mathrm{int}}^{(f)}=\mu_{B} g_{\perp} \hat{B} \Lambda_{f} \sum_{m} \sqrt{2-m(m+1)} Q_{f}^{m+1, m}
$$

We have introduced quadrature operators $Q_{f}^{m+1, m}$ as the real (Re) or imaginary (Im) parts of the corresponding Hubbard operators such that

$$
Q_{f}^{m+1, m}= \begin{cases}\operatorname{Re}\left(X_{f}^{m+1, m}\right), & f=1,2 ; \\ \operatorname{Im}\left(X_{f}^{m+1, m}\right), & f=3,4 .\end{cases}
$$

The orientation parameter $\Lambda_{f}=\sin \theta$, with $\theta=35.3^{\circ}$ for $f=1,2$, and $\Lambda_{f}=1$ for $f=3,4$.

In every local quantization axis- $f$ system, there is $\mathrm{SU}(3)$ symmetry of the spin-1 ensembles that can be represented by the local Hubbard operators associated with the generators of the Lie algebra. We can exploit existence of three spin-1/2 sub-groups by choosing the Gell-Mann fundamental representation of the SU(3) Lie 
algebra. These three spin- $1 / 2$ groups are called as isospin (T), U-spin and V-spin. In terms of Hubbard operators, the spin- $1 / 2$ operators can be defined to be

$$
\begin{aligned}
& V_{f}^{-}=X_{f}^{-0}, V_{f}^{+}=X_{f}^{0-}, V_{f}^{3}=\frac{1}{2}\left(X_{f}^{00}-X_{f}^{--}\right) \\
& U_{f}^{-}=X_{f}^{0+}, U_{f}^{+}=X_{f}^{+0}, U_{f}^{3}=\frac{1}{2}\left(X_{f}^{++}-X_{f}^{00}\right), \\
& T_{f}^{-}=X_{f}^{-+}, T_{f}^{+}=X_{f}^{+-}, T_{f}^{3}=\frac{1}{2}\left(X_{f}^{++}-X_{f}^{--}\right) .
\end{aligned}
$$

Each subgroup obeys the usual $\mathrm{SU}(2)$ algebra with $\left[L_{-}, L_{+}\right]=-2 L_{3}, L=U, V, T$. In addition, the subgroups are coupled to each other through the commutation relations such as $\left[V_{f}^{+}, U_{f}^{+}\right]=-T_{f}^{+}$. Following analogies with excitons and magnons, we can attempt to contract the sub-spin SU(2) algebras to Weyl-Heisenberg algebra. When most of the N-V centers remain in the ground singlet level, weak excitations to the upper levels can be interpreted as quasi-spin waves. In the low excitation and large $N$ limit, we can write $V_{3} \approx N / 2$ and $U_{3} \approx-N / 2$. Hence with the contraction parameter $1 / \sqrt{N}$, we can introduce approximately bosonic operators as $v_{f}=V_{f}^{+} / \sqrt{N}$ and $u_{f}=U_{f}^{-} / \sqrt{N}$ so that $\left[v_{f}, v_{f}^{\dagger}\right]=1,\left[v_{f}, v_{f}\right]=0$, and same for $u_{f}$. Clearly, the spin- $1 / 2$ sub-groups are also uncoupled from each other in this limiting case as the isospin subgroup would be weakly populated relative to $N$. Provided that the interaction Hamiltonian has no explicit dependence to the isospin generators, through some additional coupling mechanism between $|X\rangle$ and $|Y\rangle$, one can ignore the invariant isospin subspace and trace the dynamics in the closed $h_{2}$ subspace of the contracted $S U(2) \bar{\otimes} h_{2}$.

Introducing the replacements $v_{1,2}=-\mathrm{ia}_{1,2}, \mathrm{u}_{1,2}=\mathrm{ib}_{1,2}$ and $v_{3,4}=a_{3,4}, u_{3,4}=b_{3,4}$, the bosonic Hamiltonian can be compactly expressed to be

$$
H=\omega_{c} a_{c}^{\dagger} a_{c}+\sum_{f}\left(H_{0}^{(f)}+H_{\mathrm{int}}^{(f)}\right)
$$

where

$$
\begin{aligned}
& H_{0}^{(f)}=D\left(a_{f}^{\dagger} a_{f}+b_{f}^{\dagger} b_{f}\right) \\
& H_{\mathrm{int}}^{(f)}=-i \sqrt{N_{f}^{\prime}} \mu_{B} g_{\perp} \hat{B}\left(a_{f}-a_{f}^{\dagger}+b_{f}^{\dagger}-b_{f}\right) .
\end{aligned}
$$

We define an effective number of N-V centers in a particular crystallographic class as $N_{f}^{\prime}=N_{f} \Lambda_{f}^{2}$. It can be shown that the system posses a "dark" mode, described by $d_{f}=\left(a_{f}+b_{f}\right) / \sqrt{2}$, uncoupled from the cavity mode. The Hamiltonian can be expressed solely in terms of the bright $c_{f}=\left(a_{f}-b_{f}\right) / \sqrt{2}$ modes as

$$
\begin{aligned}
& H_{0}^{(f)}=D c_{f}^{\dagger} c_{f}, \\
& H_{\mathrm{int}}^{(f)}=-i \sqrt{N_{f}^{\prime}} \mu_{B} g_{\perp} \hat{B}\left(c_{f}-c_{f}^{\dagger}\right) .
\end{aligned}
$$

We now seek similar dark modes in the total Hamiltonian, exploting the similar magnetic response of pairs of crystallographic groups $(1,2)$ and $(3,4)$. We find the corresponding dark modes $d_{12}$ and $d_{34}$ to be

$$
\begin{aligned}
& d_{12}=\frac{\sqrt{N_{2}^{\prime}} c_{1}-\sqrt{N_{1}^{\prime}} c_{2}}{\sqrt{N_{1}^{\prime}+N_{2}^{\prime}}}, \\
& d_{34}=\frac{\sqrt{N_{4}^{\prime}} c_{3}-\sqrt{N_{3}^{\prime}} c_{4}}{\sqrt{N_{3}^{\prime}+N_{4}^{\prime}}} .
\end{aligned}
$$

The corresponding bright modes $c_{12}$ and $c_{34}$ becomes

$$
c_{12}=\frac{\sqrt{N_{1}^{\prime}} c_{1}+\sqrt{N_{2}^{\prime}} c_{2}}{\sqrt{N_{1}^{\prime}+N_{2}^{\prime}}}
$$




$$
c_{34}=\frac{\sqrt{N_{3}^{\prime}} c_{3}+\sqrt{N_{4}^{\prime}} c_{4}}{\sqrt{N_{3}^{\prime}+N_{4}^{\prime}}}
$$

Continuing with that strategy, we finally find the collective dark mode $d_{N V}$ and the bright mode $c_{N V}$ of the $\mathrm{N}-\mathrm{V}$ centers of different crystallographic classes such that

$$
\begin{aligned}
& d_{N V}=\frac{\sqrt{N_{3}^{\prime}+N_{4}^{\prime}} c_{12}-\sqrt{N_{1}^{\prime}+N_{2}^{\prime}} c_{34}}{\sqrt{N^{\prime}}}, \\
& c_{N V}=\frac{\sqrt{N_{1}^{\prime}+N_{2}^{\prime}} c_{12}+\sqrt{N_{3}^{\prime}+N_{4}^{\prime}} c_{34}}{\sqrt{N^{\prime}}},
\end{aligned}
$$

where an effective total number of $\mathrm{N}-\mathrm{V}$ centers is defined to be

$$
N^{\prime}=\sum_{f} N_{f}^{\prime}=\left(N_{1}+N_{2}\right) \sin ^{2} \theta+N_{3}+N_{4} .
$$

The given form of $c_{N V}$ emphasizes the pairwise grouping of equivalent classes $(1,2)$ and $(3,4)$. In terms of the single classes, the expression simplifies to

$$
c_{N V}=\frac{1}{N^{\prime}} \sum_{f} \sqrt{N_{f}} \Lambda_{f} c_{f} .
$$

If $N$ is sufficiently large, it may be assumed that $N_{f}=N / 4$. In that case these expressions are greatly simplified, and becomes independent of the number of $\mathrm{N}-\mathrm{V}$ centers such that

$$
\begin{aligned}
d_{N V} & =\frac{1}{\sqrt{2\left(1+\sin ^{2} \theta\right)}}\left[c_{1}+c_{2}-\sin \theta\left(c_{3}+c_{4}\right)\right], \\
c_{N V} & =\frac{1}{\sqrt{2\left(1+\sin ^{2} \theta\right)}}\left[\left(c_{1}+c_{2}\right) \sin \theta+c_{3}+c_{4}\right] .
\end{aligned}
$$

In terms of the quasi-spin wave operators we get, for the $c_{N V}$,

$$
\begin{aligned}
c_{N V} & =\frac{1}{2 \sqrt{\left(1+\sin ^{2} \theta\right)}}\left[\left(v_{1}+v_{2}+u_{1}+u_{2}\right) i \sin \theta\right. \\
& \left.+v_{3}+v_{4}-u_{3}-u_{4}\right] .
\end{aligned}
$$

Let us now consider two N-V center ensembles, (labeled by A and B), placed at the adjacent antinodes in the cavity magnetic field. In that case the total Hamiltonian can be written in terms of the bright N-V center mode operators described above. Introducing $a, b$ operators, replacing the $c_{N V}^{A, B}$ of the corresponding spin ensemble, we find

$$
\begin{aligned}
H & =H_{0}+H_{1} \\
H_{0} & =D\left(a^{\dagger} a+b^{\dagger} b\right)+\omega_{c} a_{c}^{\dagger} a_{c} \\
H_{1} & =\frac{\mu_{B} B_{0} g_{\perp}}{\sqrt{2}}\left(a_{c}+a_{c}^{\dagger}\right)\left[\sqrt{N_{A}^{\prime}}\left(a-a^{\dagger}\right)-\sqrt{N_{B}^{\prime}}\left(b-b^{\dagger}\right)\right] .
\end{aligned}
$$

It is essential to realize that even if the ensembles are identically prepared with the same number of defects, it is not guaranteed that same number of $\mathrm{N}-\mathrm{V}$ centers can be found in the same crystallographic classes of the two ensembles. Thus, we cannot assume the effective number of $\mathrm{N}-\mathrm{V}$ centers, $N_{A, B}^{\prime}$ are the same.

The Hamiltonian can be viewed in a frame rotating by $\omega_{c}$ by making a quantum analogue of classical non-inertial frame transformation via employing a unitary transformation of $U=\exp \left[-i \omega_{c} t\left(a^{\dagger} a+b^{\dagger} b+a_{c}^{\dagger} a_{c}\right)\right]$. 
Subsequently dropping the rapidly rotating terms in the so called Rotating Wave Approximation (RWA) the Hamiltonian is reduced to

$$
\begin{aligned}
& H_{0}=\Delta\left(a^{\dagger} a+b^{\dagger} b\right), \\
& H_{1}=g\left[a_{c}^{\dagger}\left(\sqrt{N_{B}^{\prime}} b-\sqrt{N_{A}^{\prime}} a\right)+H . c .\right],
\end{aligned}
$$

where the coupling constant $g=\mu_{B} g_{\perp} B_{0} / \sqrt{2}$ and detuning parameter $\Delta=D-\omega_{c}$ is introduced.

We can now utilize a final dark mode $d$ and bright mode $c$ transformation, using

$$
\begin{aligned}
& d=\frac{\sqrt{N_{A}^{\prime}} b+\sqrt{N_{B}^{\prime}} a}{\sqrt{N_{A}^{\prime}+N_{B}^{\prime}}}, \\
& c=\frac{\sqrt{N_{B}^{\prime}} b-\sqrt{N_{A}^{\prime}} a}{\sqrt{N_{A}^{\prime}+N_{B}^{\prime}}},
\end{aligned}
$$

to transform the total Hamiltonian into an exciton-polariton model in the form

$$
H=\Delta c^{\dagger} c+g \sqrt{N^{\prime}}\left(a_{c}^{\dagger} c+a_{c} c^{\dagger}\right]
$$

where $N^{\prime}=N_{A}^{\prime}+N_{B}^{\prime}$. The Hamiltonian is in a quadratic form that can be diagonalized by the usual Bogoliubov transformation. This allows for writing the Hamiltonian in terms of N-V polariton operators $p_{\lambda}$ such that $H=\sum_{\lambda} E_{\lambda} p_{\lambda}^{\dagger} p_{\lambda}$ with $\lambda= \pm$ are the labels for the upper and lower $\mathrm{N}-\mathrm{V}$ polariton branches $E_{\lambda}$. Considering a network of coplanar waveguide resonators labeled by $j=1 . . M$, coupled through microwave photon exchange, Hamiltonian for such a network could be written as

$$
H=\sum_{j \lambda} E_{j \lambda} p_{j \lambda}^{\dagger} p_{j \lambda}+\sum_{j j^{\prime} \lambda \lambda^{\prime}} t_{j j^{\prime}}^{\lambda^{\prime} \lambda} p_{j \lambda}^{\dagger} p_{j^{\prime} \lambda^{\prime}}
$$

with $t_{j j^{\prime}}^{\lambda^{\prime} \lambda}$ is tunneling coefficient. This Hamiltonian describes a generalized continuous-time quantum random walk. ${ }^{34}$ For the case of single polariton branch, it is equivalent to recently realized quantum walks of photons in coupled waveguide arrays. ${ }^{35}$

Alternatively, we can also consider dispersive regime of circuit QED, $|\Delta| \gg g \sqrt{N^{\prime}}$ and eliminate the cavity field using Fröchlich polaron transformation to put the Hamiltonian (44) in an effective form

$$
H_{\mathrm{eff}}=\sum_{f} \mu_{f} c_{f}^{\dagger} c_{f}+\sum_{f \neq f^{\prime}} J_{f f^{\prime}} c_{f}^{\dagger} c_{f}^{\prime},
$$

where the effective free energy is $\mu_{f}=-g^{2} \Lambda_{f}^{2} N_{f} / 2 \Delta$ and hopping coefficients are $J_{f f^{\prime}}=-g^{2} \Lambda_{f} \Lambda_{f^{\prime}} \sqrt{N_{f} N_{f^{\prime}}} / 2 \Delta$. This form of Hamiltonian suggests Rabi oscillations and periodic quantum state transfers between crystallographic groups. Which groups to be involved can be chosen by using a bias magnetic field. It should be clear from above formalism that this form could be valid for two spatially distinct $\mathrm{N}-\mathrm{V}$ center ensembles as well. In particular using a bias magnetic field to select a particular crystallographic class, Rabi oscillations and periodic state transfer would be analogously realized for such two spatially distinct N-V center ensembles. This Hamiltonian in the dispersive regime could also be further diagonalized again in terms of Bogoluibov quasi-particles. It is known that such a model yields mode entanglement between its consitituent modes of the subsystems. In our case this suggests that crystallographic classes would be mode entangled among each other. Depending on the developments of addressing each $\mathrm{N}-\mathrm{V}$ class separately for controllable population building up in each of them, one can consider using classes (12) and (34) in a single diamond crystal as spin-ensemble qubit. Bias field can be used to select which classes to be entangled. Furthermore, incase of two spatially distinct N-V center ensembles, using bias field to select a single $\mathrm{N}-\mathrm{V}$ class for each of them yields mode entanglement of such spatially separated $\mathrm{N}-\mathrm{V}$ ensembles.

Finally, the form of $J_{f f^{\prime}}$ suggests that in addition to geometric factors $\Lambda_{f}$, period of the Rabi oscillations between crystallographic classes depends on their populations. Thus measurement of various time dependent fidelities of the state transfer between such classes would yield information on the population distribution of N-V centers among their classes. 


\section{CONCLUSION}

Summarizing, we have examined the two N-V center ensembles placed in a CPWG cavity. All the crystallographic classes of the $\mathrm{N}-\mathrm{V}$ centers are taken into account in the analysis. By recognizing a series of invariant subspaces in the total Hilbert space, the dynamics of the system is completely specified by introducing the collective N-V center-polaritons. Such a model can be utilized for quantum random walk demonstration in a network of N$\mathrm{V}$ centers in diamond crystals coupled to superconduciing coplanar waveguide resonators. The state transfer and mode entanglement between two distant $\mathrm{N}-\mathrm{V}$ center ensembles as well as between distinct crystallographic groups in a single diamond crystal are revealed by using Fröchlich transformation for the cavity field mediated interaction between the spin ensembles. Effects of geometric factors and populations on the Rabi oscillations and fidelities of the state transfer are found.

\section{ACKNOWLEDGMENTS}

Ö.E.M. gratefully acknowledges Atac Imamoğlu and E. Ilgunsatiroglu for many illuminating discussions. This work is supported by D.P.T. (T.R. Prime Ministry State Planning Organization) under Project No. 2009K120200.

\section{REFERENCES}

[1] Wu, H., George, R. E., Wesenberg, J. H., Mølmer, K., Schuster, D. I., Schoelkopf, R. J., Itoh, K. M., Ardavan, A., Morton, J. J. L., and Briggs, G. A. D., "Storage of multiple coherent microwave excitations in an electron spin ensemble," Phys. Rev. Lett 105, 140503 (2010).

[2] Schuster, D. I., Sears, A. P., Ginossar, E., DiCarlo, L., Frunzio, L., Morton, J. J. L., Wu, H., Briggs, G. A. D., Buckley, B. B., Awschalom, D. D., and Schoelkopf, R. J., "High-cooperativity coupling of electronspin ensembles to superconducting cavities," Phys. Rev. Lett. 105, 140501 (2010).

[3] Kubo, Y., Ong, F. R., Bertet, P., Vion, D., Jacques, V., Zheng, D., Dréau, A., Roch, J.-F., Auffeves, A., Jelezko, F., Wrachtrup, J., Barthe, M. F., Bergonzo, P., and Esteve, D., "Strong coupling of a spin ensemble to a superconducting resonator," Phys. Rev. Lett 105, 140502 (2010).

[4] Wallquist, M., Hammerer, K., Rabl, P., Lukin, M., and Zoller, P., "Hybrid quantum devices and quantum engineering," Phys. Scr. T137, 014001 (2009).

[5] Müstecaplıoğlu, Ö. E., "Quantum coherence and correlations of optical radiation by atomic ensembles interacting with a two-level atom in a microwave cavity," Phys. Rev. A 83, 023805 (2011).

[6] Blais, A., Huang, R.-S., Wallraff, A., Girvin, S. M., and Schoelkopf, R. J., "Cavity quantum electrodynamics for superconducting electrical circuits: An architecture for quantum computation," Phys. Rev. A 69, 062320 (2004).

[7] Wallraff, A., Schuster, D. I., Blais, A., Frunzio, L., Huang, R.-S., Majer, J., Kumar, S., Girvin, S. M., and Schoelkopf, R. J., "Strong coupling of a single photon to a superconducting qubit using circuit quantum electrodynamics," Nature (London) 431, 162-167 (2004).

[8] Schuster, D. I., Houck, A. A., Schreier, J. A., Wallraff, A., Gambetta, J. M., Blais, A., Frunzio, L., Majer, J., Johnson, B. R., Devoret, M. H., Girvin, S. M., and Schoelkopf, R. J., "Resolving photon number states in a superconducting circuit," Nature (London) 445, 515-518 (2007).

[9] Simons, R. N., [Coplanar Waveguide Circuits, Components, and Systems], John Wiley and Sons, Inc. (2001).

[10] Some typical commercial FEM solvers that can be suitable for microwave transmission line resonator designs can be HFSS (TM) of Ansoft, Microwave Studio (TM) of CST or COMSOL (TM) of COMSOL Group.

[11] Bucks, E. and Yurke, B., "Dephasing due to intermode coupling in superconducting stripline resonators," Phys. Rev. A 73, 023815 (2006).

[12] Dahm, T. and Scalapino, D. J., "Theory of intermodulation in a superconducting microstrip resonator," J. Appl. Phys. 81, 2002-2009 (1997).

[13] Rowe, D. A. and Lao, B. Y., "Numerical analysis of shielded coplanar waveguides," IEEE Trans. Microwave Theory Tech. 31, 911-915 (1983).

[14] Yurke, B. and Denker, J. S., "Quantum network theory," Phys. Rev. A 29, 1419-1437 (1984).

[15] Devoret, M. H., "Quantum fluctuations in electrical circuits," in [Quantum Fluctuations: Les Houches, Session LXIII], Reynaud, S., Giacobino, E., and Zinn-Justin, J., eds., 351, Elsevier, Amsterdam (1997). 
[16] Burkard, G., Koch, R. H., and Vincenzo, D. P., "Multilevel quantum description of decoherence in superconducting qubits," Phys. Rev. B 69, 064503 (2004).

[17] Weber, J., "Quantum theory of a damped electrical oscillator and noise. ii. the radiation resistance," Phys. Rev. 94, 211-214 (1954).

[18] Stevens, K. W. H., "The wave mechanical damped harmonic oscillator," Proc. Phys. Soc. 62, 1027 (1958).

[19] Stevens, K. W. H., "A comment on quantum-mechanical damping," Phys. Lett. 75, 463 (1980).

[20] Wang, Y. D. and Sun, C. P., "A lossy transmission line as a quantum open system in the standard quantum limit," Unpublished, E-print, arXiv:cond-mat/0406748 (2004).

[21] Louisell, W. H., [Quantum Statistical Properties of Radiation], John Wiley and Sons, New York (1990).

[22] Hong-Yi, F., Bao-Long, L., and Ji-Suo, W., "Number-phase quantization scheme for l-c circuit," Commun. Theor. Phys. 48, 1038-1040 (2007).

[23] Jelezko, F., Gaebel, T., Popa, I., Gruber, A., and Wrachtrup, J., "Observation of coherent oscillations in a single electron spin," Phys. Rev. Lett. 92, 076401 (2004).

[24] Manson, N. B., Harrison, J. P., and Sellars, M. J., "Nitrogen-vacancy center in diamond: Model of the electronic structure and associated dynamics," Phys. Rev. B 74, 104303 (2006).

[25] Epstein, R. J., Mendoza, F. M., Kato, Y. K., and Awschalom, D. D., "Anisotropic interactions of a single spin and dark-spin spectroscopy in diamond," Nature Physics 1, 94-98 (2005).

[26] Hanson, R., Dobrovitski, V. V., Feiguin, A. E., Gywat, O., and Awschalom, D. D., "Coherent dynamics of a single spin interacting with an adjustable spin bath," Science 320, 352-355 (2008).

[27] Jelezko, F., Gaebel, T., Popa, I., Domhan, M., Gruber, A., and J.Wrachtrup, "Observation of coherent oscillation of a single nuclear spin and realization of a two-qubit conditional quantum gate," Phys. Rev. Lett. 93, 130501 (2004).

[28] Childress, L., Dutt, M. V. G., Taylor, J. M., Zibrov, A. S., Jelezko, F., Wrachtrup, J., Hemmer, P. R., and Lukin, M. D., "Coherent dynamics of coupled electron and nuclear spin qubits in diamond," Science 314, $281-285$ (2006).

[29] van Oort, E., Manson, N. B., and Glasbeek, M., "Optically detected spin coherence of the diamond n-v centre in its triplet ground state," J. Phys. C 21, 4385-4391 (1988).

[30] Redman, D. A., Brown, S., Sands, R. H., and Rand, S. C., "Spin dynamics and electronic states of n-v centers in diamond by epr and four-wave-mixing spectroscopy," Phys. Rev. Lett 67, 3420 (1991).

[31] Charnock, F. T. and Kennedy, T. A., "Combined optical and microwave approach for performing quantum spin operations on the nitrogen-vacancy center in diamond," Phys. Rev. B 64, 041201(R) (2001).

[32] Taylor, J. M., Cappellaro, P., Childress, L., Jiang, L., Budker, D., Hemmer, P. R., Yacoby, A., Walsworth, R., and Lukin, M. D., "High-sensitivity diamond magnetometer with nanoscale resolution," Nature Physics 4, 810-816 (2008).

[33] Lai, N. D., Zheng, D., Jelezko, F., Treussart, F., and Roch, J.-F., "Influence of a static magnetic field on the photoluminescence of an ensemble of nitrogen-vacancy color centers in a diamond single-crystal," Unpublished, E-print, arXiv:cond-mat/0908.1327v2 (September 2009).

[34] Kempe, J., "Quantum random walks: An introductory overview," Contemp. Phys. 44, 307-327 (2003).

[35] Peruzzo, A., Lobino, M., Matthews, J. C. F., Matsuda, N., Politi, A., Poulios, K., Zhou, X.-Q., Lahini, Y., Ismail, N., Wörhoff, K., Bromberg, Y., Silberberg, Y., Thompson, M. G., and OBrien, J. L., "Quantum walks of correlated photons," Science 329, 1500-1503 (2010). 